Methods Single endoscopist using ESD and Hybrid ESD (H-ESD) technique was retrospectively audited from April 2004 to August 2012. Service evaluation data of 38 patients with large sessile polyps who underwent ESD and Hybrid ESD was reviewed from a cohort of 224 colonoscopies referred for large polyp EMR. All procedures were intended as ESD. NICE recommendations for case selection were followed in $92 \%$ cases. Due to challenges in submucosal dissection of the large lesions, piecemeal resection was done after circumferential cutting. First follow-up endoscopy was performed at 3-6 months and the second at 12-14 months.

Results Mean age was 70 with 16 males and 22 females. Mean size of polyp in ESD group was $26 \mathrm{~mm}$. Range $15-50 \mathrm{~mm}$. Mean size in the H-ESD group was $49 \mathrm{~mm}$. Range $20-100 \mathrm{~mm}$. Complete resections were achieved in 17 (44\%) out of 38 cases. Due to piecemeal resection pathologists were not able to confirm adequacy of excision in 12 cases. In 9 cases resection was reported incomplete on index procedure. ESD performed in 13 (34\%) cases. Complete resection achieved in 6 . Out of 7 incomplete resections in the ESD group, 3 were reported by pathologists as lesion extending to the lateral margin hence incomplete excision. Histology did not comment on margin clearance in 3 ESD. 1 ESD was a sub mucosal lipoma on histology. This was an unintentional ESD for lipoma. Histology: ESD group: TVA with LGD 7, TVA with HGD 5. H-ESD was performed in $25(65.7 \%)$ cases. Complete resection achieved in 11 cases, incomplete resection in 7 and lateral margin clearance not confirmed in $7 \mathrm{H}$-ESD cases due to piecemeal resection. Histology: H-ESD group: TVA with LGD 16, TVA with HGD 6 and adenocarcinoma in 2 cases-one's lateral and deep margins were clear and the other was incomplete and referred to MDT In $4 \mathrm{ESD}$ and $7 \mathrm{H}-\mathrm{ESD}$ cases there was minor bleeding controlled endoscopically at the time. 1 delayed post- H-ESD bleeding required 11 days of hospital stay and 2 units of blood transfusion. 1 retroperitoneal perforation and 1 case of serosal cut managed conservatively with clips and antibiotics. APC performed in 16 $(42 \%)$ out of 38 . Recurrence was identified in $6 \mathrm{H}$-ESD cases (15.7\%). 5 local recurrences detected at 3 months and 1 local recurrence detected at 24 months. In 13 ESD cases no perforation or recurrence upto 14 months was noted despite 5 histologically incomplete dissections.

Conclusion ESD in bowel is challenging and has a long learning curve. These procedures should be performed by trained endoscopists in accredited units and a national registry should be maintained.

Disclosure of Interest None Declared.

\section{PTH-062 ENDOSCOPIC MUCOSAL RESECTION OF LARGE COLORECTAL POLYPS IN NON SCREENING COLONOSCOPIES HAS A HIGH RECURRENCE RATE:}

doi:10.1136/gutjnl-2013-304907.549

1,"S M Ali, 'R Kalla, 'M Hendrickse. 'Gastroenterology, Blackpool Victoria Hospital, Blackpool, UK

Introduction EMR of large colorectal polyps has been reported to be a safe and effective technique in UK. Although the success of large polyp EMR has been reported within regional bowel cancer screening centres, there is a paucity of data comparing outcomes between screening and non-screening centres.

Methods A total of 61 screening and 60 non-screening EMR of polyps $2 \mathrm{~cm}$ or greater were performed from January 2008 to December 2011. Data was collected from 3 hospitals for screening EMR and 1 district hospital for non-screening EMR

Results The table below summarises the findings at initial EMR for both groups.

\section{Abstract PTH-062 Table 1}

\begin{tabular}{lcc}
\hline Characteristics & $\begin{array}{c}\text { Results for Screening } \\
\text { group n (\%) }\end{array}$ & $\begin{array}{c}\text { Results for non-screening } \\
\text { group } \mathbf{n}(\%)\end{array}$ \\
\hline Number of patients & 61 & 60 \\
Mean size (in mm) & 35 & 43 \\
Location (Left colon) & $43(70)$ & $36(60)$ \\
Histology (Low Grade Dysplasia) & $47(83)$ & $36(67)$ \\
Histology (High Grade Dysplasia) & $7(12)$ & $11(20)$ \\
Histology (Adenocarcinoma) & $3(5)$ & $7(13)$ \\
Bleeding Complication & $2(3)$ & $3(5)$ \\
Perforation & $0(0)$ & $1(2)$ \\
\hline
\end{tabular}

Surveillance data was available for 37 patients in the non-screening group compared to all 61 patients in the screening group up-to 3 months post EMR. Number of recurrences were $6(10 \%)$ with mean polyp size of $25 \mathrm{~mm}$ in the screening group compared to $10(27 \%)$ in the non screening group with mean polyp size of $50 \mathrm{~mm}$.

All recurrences in both groups were resected and complete. Histology confirmed low grade dysplasia (LGD) in $80 \%$, while 2 patients had evidence of high grade dysplasia (HGD) in the non-screening group. All showed LGD in the screening group. At 12 months surveillance post EMR, 43 screening patients had follow-up data with 8 recurrences detected compared to 14 in the non screening group with 4 recurrences.

Conclusion Endoscopic mucosal resection of polyps in screening group appears to be safer with low complication rates and low recurrence rate up to 12 months post EMR compared to the nonscreening groups. This may be due to a multitude of factors such as patient age, co-morbidites and the characteristics of polyps. Clinical features of patients undergoing EMR were similar in both groups. There was a trend to higher recurrence and complication rate in the non screening group. The follow up post EMR was less consistent in the non screening group possibly due to less stringent protocols for follow up. This may have affected the surveillance and recurrence rates.

Disclosure of Interest None Declared.

\section{REFERENCES}

Lim TR, Mahesh V, Singh S, et al. Endoscopic mucosal resection of colorectal polyps in typical UK hospitals. World J Gastroenterol.2010; 16(42): 5324-8.

Boix J, Lorenzo-Zúñiga V, Moreno de Vega V, et al. Endoscopic removal of large sessile colorectal adenomas: is it safe and effective? Dig Dis Sci 2007; 52 840-844

Salama M, Ormonde D, Quach T, et al. Outcomes of endoscopic resection of large colorectal neoplasms: an Australian experience. J Gastroenterol Hepatol 2010; 25 $84-89$

\section{PTH-063 ESTABLISHING AUDIT STANDARDS FOR COLONIC STENT INSERTION WILL FACILITATE SERVICE EVALUATION AND PLANNING FOR A RAPIDLY GROWING SERVICE}

doi:10.1136/gutjnl-2013-304907.550

1,*T M Cacciottolo, ' $\mathrm{D}$ Menzies, 'A Shenoy, 'D 0'Riordan, 'I Gooding. 'Gastroenterology; ${ }^{2}$ Surgery, Colchester General Hospital, Colchester, UK

Introduction Self-expandable metal stent (SEM) placement is the recommended treatment option by the National Institute for Clinical Excellence (NICE) for acute colorectal obstruction in the palliative management of inoperable colorectal cancer (CRC) as well as a bridge to planned single stage surgical intervention[1]. NICE guidance recommends that centres offering this treatment modality should have teams with expertise and capacity to stent 15 people per million population per annum[2]. It does not however provide any suitable standard for audit. 
Methods All colonic stents inserted between $1^{\text {st }}$ January 2008 and $31^{\text {st }}$ December 2011 were included. Patient characteristics, procedure related data, outcomes and survival were recorded retrospectively from health records.

Results Systematic review of 29 case series of stent insertions reported a clinical success rate of $88 \%, 10 \%$ stent migration rate, $4 \%$ perforation rate, $10 \%$ reobstruction rate and $1 \%$ procedure-related mortality rate[3]. These were applied as our audit standards.

A total of 69 colonic stents (in 64 patients, 35 male, mean age 74.2 years) were inserted. 15 stents were inserted in 2008 and this increased steadily to 25 in 2011. The most common indication for colonic stent insertion was a malignant stricture $(89.9 \%)$. There was a $98.5 \%$ technical success rate, $2.9 \%$ migration rate, $7.2 \%$ perforation rate and a $4.3 \%$ reobstruction rate within one month of the procedure. The clinical success rate, ie successful stent insertion and decompression within 96 hours, was $88.4 \%$.

$61.1 \%$ had stent insertion as a bridge to surgery. Mean survival post procedure was 200 days (range 1-779 days). The were 2 deaths as a direct consequence of the procedure $(2.9 \%)$, both of which followed perforation in patients who were too frail to undergo surgery. Conclusion We have defined and applied audit standards for colonic stent insertion. Success and complication rates at our hospital compare favourably to published rates. There is a steady increase in colonic stent insertions and in our 2011 cohort this equated to 67.6 patients per million per annum. It is likely that this number will continue to increase and endoscopy units should take this into account when planning their service provision. Audit support will be essential, and we encourage the adoption of consistent audit standards to facilitate comparison between units.

\section{Disclosure of Interest None Declared.}

\section{REFERENCES}

1. National Institute for Health and Clinical Excellence. (2004). Improving Outcomes in Colorectal Cancers. CSGCC.

2. National Institute for Health and Clinical Excellence.(2010). Improving Outcomes in Colorectal Cancers. Manual Update.

3. Khot et al. Systematic Review of the Safety and Efficacy of Colorectal Stents. BJS 2002(89):1096:1102

\section{PTH-064 NARROW BAND IMAGING FACILITATES DETECTION OF INLET PATCHES IN THE CERVICAL OESOPHAGUS}

doi:10.1136/gutjnl-2013-304907.551

'S Al-Mammari, 1," U Selvarajah, ' $\mathrm{J}$ E East, ${ }^{1}$ A A Bailey, 'B Braden. 'Translational Gastroenterology Unit, Oxford University Hospitals Trust, Oxford, UK

Introduction Ectopic gastric mucosa in the cervical oesophagus (inlet patch) is easily missed on endoscopic examination because of its localization, usually just below the upper oesophageal sphincter. In some individuals, an inlet patch might cause symptoms such as dysphagia, globus sensation, odynophagia or coughing.

We prospectively investigated whether narrow band imaging (NBI) during endoscopic inspection of the cervical oesophagus improves the visualisation of heterotopic gastric mucosa and increases the detection rate of inlet patches.

Methods Subsequent upper gastrointestinal endoscopies were performed by three experienced endoscopists using videoendoscopes with NBI option (Olympus Lucera Spectrum system). Between $01 / 02 / 2010$ and $01 / 12 / 2011$, patients with various indications for upper gastrointestinal endoscopy were consecutively assigned to the lists of three specialists in endoscopy. One endoscopist routinely applied NBI during extubation of the endoscope (NBI) in addition to standard white light endoscopy, the second was aware of the study, but did not routinely use NBI, the third was unaware of the study and did not use NBI. The presence of an inlet patch was documented by photo imaging.
Results 1407 upper endoscopies were performed. When NBI was used during extubation of the oesophagus, inlet patches were detected in 17 out of 510 endoscopies. The detection rate of inlet patches using NBI (3.3\%) was significantly higher than in conventional white light endoscopy only (10/897; $1.1 \% ; p=0.007)$, whether the endoscopist was aware $(4 / 382 ; 1.1 \% ; p=0.026)$ or unaware of the study $(6 / 515 ; 1.2 \% ; p=0.02$. $)$. Using NBI, the relative chance to detect an inlet patch increases about threefold (odds ratio 3.05 , 95\% CI 1.39-6.71).

Conclusion Withdrawal of the endoscope from the cervical oesophagus using narrow band imaging increased the detection rate of inlet patches about threefold compared to standard white light endoscopy. NBI may assist in the accurate assessment of patients presenting with globus sensation, dysphagia or chronic cough, to identify rare causes such as an inlet patch that may be amenable to therapy.

Disclosure of Interest None Declared.

\section{PTH-065 DIAGNOSTIC AND THERAPEUTIC UTILITY OF SPYGLASS SINGLE OPERATOR PERORAL CHOLANGIOSCOPY FOR INDETERMINATE BILIARY LESIONS: A SINGLE CENTRE EXPERIENCE IN SCOTLAND}

doi:10.1136/gutjnl-2013-304907.552

1." U Basavaraju, 'A Fraser, 'J Leeds. 'Department of Gastroenterology, Aberdeen Royal Infirmary, Aberdeen, UK

Introduction The SpyGlass single-operator peroral cholangioscopy is a promising technique designed to overcome some of the limitations of conventional peroral cholangioscopy. We aimed to evaluate the diagnostic utility of the SpyGlass system in a cohort of patients with indeterminate biliary strictures and lesions.

Methods All patients who were listed for SpyGlass cholangioscopy for for further evaluation or treatment of indeterminate strictures or filling defects previously identified at ERCP or other imaging modality were included in this study. After SpyGlass direct visual evaluation, targeted biopsies were taken with the SpyBite forceps and histopathological assessment was made by experienced gastrointestinal pathologists.

Results Between April 2009 and January 2013, 21 subjects (mean age 60, 12 males) underwent SpyGlass cholangioscopy for indeterminate biliary strictures $(n=12)$ or filling defects $(n=9)$. SpyGlass cannulation was not possible in 1 subject. In those with previously identified strictures, $8 / 12$ had a stricture identified during SpyGlass, 2 as probable malignant and 6 as benign strictures using endoscopic criteria. In those with filling defect, choledocholithiasis was identified in 7 subjects and 1 subject was found to have a villous adenoma. The cholangioscopy was reported normal in 4 subjects (19\%). SpyBite biopsies were taken in 10 subjects with histology showing inflammation ( $\mathrm{n}=5)$, atypia $(\mathrm{n}=2)$, adenoma $(\mathrm{n}=2)$ and normal $(n=1)$ giving a biopsy adequacy of $100 \%$. Overall, the SpyGlass evaluation agreed with the histopathologic evaluation of SpyBitetargeted biopsies in $8 / 10(80 \%)$ cases and therefore a definitive diagnosis was made in $18 / 21(86 \%)$ patients. There were no serious complications with only 1 case of mild pancreatitis, 1 case of liver abscess (after 2 weeks) and 6/15 patients received prophylactic antibiotic. Two subjects have had successful surgery for cholangiocarcinoma and all but 1 subjects are alive at the mean follow-up period of 19 months.

Conclusion Using the SpyGlass cholangioscope, a definitive diagnosis can be made with a high accuracy in $86 \%$ of patients with indeterminate biliary lesions. This is primarily due to direct visualisation and targeted biopsy sampling. Therefore, the SpyGlass system should be considered in all patients with indeterminate biliary lesions.

Disclosure of Interest None Declared. 